instruments and magnification, and tourniquet control under regional or general anaesthesia. Wound extensions should be designed so as not to impair viability or cause joint contracture while providing adequate exposure. Obviously devitalised skin should be excised, but if there is doubt the hand should be dressed with antiseptic soaks, and raised and then reviewed 48 hours later. Bursting injuries are best managed by a similar "delayed primary" method with suture after four or five days of raising when oedema has settled. Skin grafts may be applied to most soft tissue defects, but flaps are essential when the bed contains bare bone, cartilage, or tendon. Flaps may be preferred in many instances where later reconstructive procedures on the deep structures are planned, or to provide sensation at key points in the hand. Selection of the most appropriate flap for a particular defect may be difficult.

Vascular impairment which does not immediately respond to skeletal stabilisation requires urgent exploration. Microvascular reconstruction may be necessary to salvage an otherwise normal digit. If the part has been severed the decision to replant or amputate should be made after detailed examination of the amputated part under the microscope. In general, injuries which always warrant replantation include any digit in children and the thumb and multiple digits in adults. At the time of injury the amputated part should be placed in a clean, dry plastic bag inside a second bag containing ice before the patient is transferred to a replantation centre.

Though many fractures in the hand may be treated conservatively, internal fixation may be indicated when the fracture is unstable, when a joint surface is damaged, and when there are vascular injuries. These techniques should offer the advantages of accurate reduction and rigid fixation allowing immediate postoperative mobilisation. In the past primary tendon repair gave poor results in the area known as "no man's land," where both finger flexors run in a fibroosseus tunnel. Delayed tendon grafting was preferred. Refined surgical technique based on more detailed anatomical knowledge, combined with dynamic traction ${ }^{5}$ or controlled passive mobilisation during the postoperative phase maintaining tendon gliding, has so improved the functional outcome of primary repair that it is now the treatment of choice. The deformities caused by damage to the extensor tendons in the finger (mallet and boutonnière) may be avoided by immediate static splintage. If exploration is undertaken soon arter injury it is usually possible to approximate divided nerves without tension. An attempt should be made to match the fascicular pattern visible at each end. The exact method of repair may be by coapting individual fascicles (single fascicular), groups of fascicles (group fascicular), or the whole nerve (epineural). There is much debate about which technique is preferable. If there is any tension, a primary or secondary nerve graft is indicated. Immediate postoperative splintage is designed to protect the healing nerve, but splinting may also be needed later to prevent any paralytic deformity becoming structural.

In young children, injuries of the fingertip may be managed conservatively, but in adults a detailed knowledge of reconstructive techniques is necessary. Length should be maintained by judicious use of local innervated flaps, advancement flaps, ${ }^{6}$ and cross finger flaps. Reconstruction or ablation of the nail bed may be needed depending on the level of injury. Skin grafts are best avoided except to cover very small defects, which will subsequently contract. Skin grafts, however, do have an important part to play in treating burns. The most important factor influencing the outcome of a burnt hand is the depth of the injury. This is difficult to assess even for those with experience. The history provides more helpful information than examination. A superficial burn heals spontaneously, deep dermal burns may need grafting, and a full thickness burn always requires at least a skin graft but often a flap cover. In such instances early surgery (within the first three to five days) may be advisable to cover exposed tendons or joints and limit wound contraction. Whatever the depth mobility must be encouraged. Plastic bags containing antiseptic liquid furnish a good occlusive dressing, which permits movement, but splinting in a protective or functional position is important at night. As with any other hand injury, raising to limit oedema is vital.

Hand surgery is a complex specialty, which requires specific training in many aspects of surgical technique. An adequate service requires a comprehensive, enthusiastic back up team of trained anaesthetists, nurses, physiotherapists, occupational therapists, splint makers, and prosthetists. Those working in accident and emergency departments must have sufficient knowledge and facilities to provide correct primary care, but undoubtedly optimal long term results will be achieved at centres specialising in and equipped for all aspects of care for the injured hand. ${ }^{7}$

Paul J SMITH

Consultant Plastic and Hand Surgeon,

Hand Clinic,

Luton and Dunstable Hospital,

Luton

BARRY M JONES

Senior Registrar in Plastic Surgery,

Regional Centre for Reconstructive Surgery,

Mount Vernon Hospital,

Northwood,

Middlesex

1 Tubiana R. Statistical studies on hand accidents. Sem Hop Paris 1979;10:403-7

2 Stack G. The medical commission on accident prevent Hand 1982;14:3-4.

3 Rank B, Wakefield A. Surgery of repair as applied to hand injuries. Baltimore:Williams and Wilkins

$\mathrm{Co}, 1953$. The tactile adherence test estimating loss of sensation after nerve injury. Hand
Harrison $\mathrm{SH}$. The 1974;6:148-9.

A leinert HE, Kutz JE, Atasoy E, Stormo A. Primary repair of flexor tendons. Orthop Clin North tazoy E, Loakimidis E, Kasdan ML, Kutz JE, Kleinert HE. Reconstruction of the amputated finger tip with a triangular volar flap. $\mathcal{F}$ Bone foint Surg 1970;52A:921-6.

Tubiana R, Gilbert A, Foucher G, Grossman JAI. The development of trcatment centres for hand and other upper extremity disorders. F Hand Surg 1983;8:654-5.

\section{Ruptured aortic aneurysm: an avoidable disaster}

Aneurysm of the abdominal aorta is relatively common: in a Scandinavian series in 1964 it was found in $2 \%$ of consecutive routine postmortem examinations.' The increasing frequency with which it is being seen by vascular surgeons reflects greater longevity, better diagnosis, wider recognition of the role of surgery, and possibly a real increase in prevalence. Operations for aneurysm have become dramatically more frequent in recent years. In Scotland, for example, they rose from 63 in 1972 to 262 in $1982 .{ }^{2}$ An operation is the appropriate treatment unless the patient has a very limited life expectancy (on account of age or illness) or the risk of operation is judged to outweigh the risk of rupture. What are these relative risks?

Untreated, an aneurysm is likely to cause early death. The 
precise risk of leaving it untreated is not known: following up an unselected population of patients with aneurysms until they rupture has not commended itself as a project. Size is the single factor which correlates best with risk of rupture. Rutherford's review of published work concluded that the five year risk of rupture in a $4 \mathrm{~cm}$ aneurysm (barely twice the normal diameter of the aorta) is less than $15 \%$, whereas at 8 $\mathrm{cm}$ it is greater than $75 \% .^{3} \mathrm{~A}$ diameter of $6 \mathrm{~cm}$ has often been quoted as a critical threshold in recommending surgery, but vascular surgeons are well aware that small aneurysms may rupture, and many do. ${ }^{4}$

In common with other arterial reconstruction operations aneurysm grafting is relatively new, the first aortic replacement being reported by Dubost and colleagues in $1952 .{ }^{5}$ Some surgeons operating today can remember machining their first bifurcation grafts out of nylon shirts. Modern Dacron prostheses are strong, reliable, and biologically inert; they function indefinitely and give rise to problems only in the rare event of their becoming infected. In comparison with those inserted for obliterative disease grafts for aneurysm are not prone to thrombosis. Suture materials have greatly improved too, and surgical techniques have become simpler and safer.

The operative mortality after elective resections of aneurysms in specialised units is now well below 5\%-which compares favourably, for example, with surgery of the colon-but the prognosis after rupture is grave. The patient may not survive to reach hospital-and we do not know how many deaths are certified in the community as due to myocardial infarction or other causes of collapse. If, however, he or she reaches hospital and lives through the admission procedures and the journey to the operating theatre the patient may then have a $50-75 \%$ chance of survival, provided that a team experienced in vascular surgery is available. ${ }^{6}$

Too many aneurysms are dealt with after they have ruptured rather than before. This message has recently been reinforced by Fielding and colleagues. ' In reporting an excellent survival rate of $57 \%$ in their series of patients with ruptured aneurysms treated surgically in Birmingham between 1960 and 1981 they noted that the proportion who had ruptured aneurysms $(36 \%)$ had not changed over the period despite the fact that most patients had had symptoms before rupture. The pattern of symptoms before rupture is not clear, and we do not know how many of their patients had actually reported the symptoms to their doctor.

Nevertheless, if a middle aged or elderly patient presents with an unexplained pain in the abdomen, flank, or back aneurysm must be considered as a possible cause. The nature, severity, and site of the pain depend on the direction in which the aneurysm expands and what structures it compresses or erodes. The pain may be continuous or intermittent. Arteriorly it may radiate to the iliac fossa or groin, usually the left. Posteriorly it is usually a midlumbar pain, which may radiate to the sacrum or mimic the commoner causes of root pain. Sometimes the patient notices a pulsating lump, but in an obese patient even a large aneurysm may be difficult to feel, and in a person of normal build it takes a practised and careful hand to distinguish between a small aneurysm and an aorta which is tortuous or pushed forward by a lordotic spine. Doctors sometimes forget that the aorta bifurcates at the level of the umbilicus, so the swelling may be felt well up in the epigastrium. A tender aneurysm suggests actual or imminent rupture and is an indication for urgent referral to a vascular surgeon.

Aortic aneurysm is a diagnosis made essentially by physical examination. A plain radiograph of the abdomen, especially a lateral view, will in most cases show the characteristic rim of calcification. If more information is needed ultrasound and CT scan will provide it. Aortography should be reserved for preoperative assessment, and not all vascular surgeons regard it as desirable even then. Others believe that the operation may be better planned if information is available on the state of the renal, mesenteric, iliac, and distal vessels.

The decision whether to recommend operation may be difficult for the patient who is infirm or elderly. It should be made in the light of the surgeon's awareness of his operative mortality and with the important help of an anaesthetist's opinion. If the surgeon is in doubt the patient may be followed up in the outpatient clinic. The development of symptoms or enlargement, measured by serial ultrasound scans, will invite a reappraisal.

The results of operating on a patient with a ruptured aneurysm may be very rewarding. The lucky survivor will return to a life which does not differ in quality or duration from that of his contemporaries in the general population. ${ }^{8}$ Nevertheless, the cost in terms of emergency and intensive care resources is great. By the time the patient had been admitted, assessed, transferred to theatre, resuscitated, anaesthetised, operated on, and, if surviving, safely installed in the intensive care unit some six hours or more will have passed. Many a team, as they toil through the night, knowing that there is a full day's work to follow, cannot but reflect how much better it would have been-for the patient and for themselves-if the operation had been done earlier in the calm and safety of a planned elective list.

Consultant Surgeon,
Royal Infirmary,
Edinburgh EH3 9YW

C VAughan Ruckley

1 Carlsson J, Sternby NH. Aortic aneurysms. Acta Chir Scand 1964;127:466-73.

Information Services Division of the Common Services Agency. Scottish hospital in-patient statistics. Annual reports 1972-82. Scottish Home and Health Department.

Rutherford RB, ed. Vascular surgen. Philadelphia: WB Saunders, 1984:755-71.

4 Darling RC. Ruptured arteriosclerotic abdominal aortic aneurysms. Am f Surg 1970;119:397-403. Dubost C, Allary M, Oeconomos N. Resection of an aneurysm of the abdominal aorta. Arch Surg 1952;64:405-8

6 Hicks GL, Eastland MW, DeWeese JA, et al. Survival improvement following aortic aneurysm surgery. Ann Surg 1975;181:863-9.

Fielding JWL, Black J, Ashton F, Slaney G. Ruptured aortic aneurysms: postoperative complications and their aetiology. Br f Surg 1984;71:487-91.

8 Sheranian L, Edwards GE, Kirklin GE. Late results in 110 patients treated by resectional replacement by aortic graft. Surg Gynecol Obstet 1959;109:309-14.

\section{A need for new converting enzyme inhibitors?}

Efficacy and freedom from side effects are the ultimate determinants of drug prescribing-and especially in hypertension, with most patients initially asymptomatic and yet requiring treatment for life. $\beta$ antagonists and diuretics used individually control blood pressure adequately in little more than half of all patients, and both of these groups of drugs have untoward effects, metabolic and symptomatic, in at least some people. Often a third drug is needed, but the same problems with efficacy and side effects leave no room for complacency. ${ }^{1}$

Captopril-the first orally active inhibitor of angiotensin converting enzyme-has contributed substantially to the 